\title{
Personalized therapeutics through pharmacogenomics
}

\section{Introduction}

Most healthcare professionals are aware of the emerging field of pharmacogenomics. Many are not aware however that it is nearing its $15^{\text {th }}$ year of pursuit to combine the science of how drugs work (pharmacology) with the science of the human genome (genomics). Although the field of pharmacogenomics is still in its infancy with only limited use in select disease areas such as cancer, cardiovascular challenges, HIV, Alzheimer's and asthma, the coordination between United States research centers is accelerating the pace and implementation in several new disease states. This approach to personalized medicine is a significant departure from previous drug development where the idea has existed that each drug works pretty much the same in every individual and where by exception over time is has been learned that many different aspects must be considered when a medication is provided to the end user.

\section{Background}

Some of the earliest efforts in applying pharmacogenomics to successful clinical therapeutics was first published in 2003 and it's utility in cancer chemotherapy. Pharmacogenomics is especially important for oncology as severe systemic toxicity and unpredictable efficacy are hallmarks of cancer therapies. The genetic polymorphisms in drug metabolizing enzymes and other molecules are responsible for much of the inter-individual differences in the efficacy and toxicity of many chemotherapy agents.

Genes are instructions, written in DNA, for building protein molecules. Different people can have different versions (slightly different DNA sequences) of the same gene. Some of these variations are relevant for health such as the tendency to develop certain diseases and are common to many different individuals.

As pharmacogenomics looks at variations in genes for proteins that influence drug responses, certain of these proteins include a number of liver enzymes that convert medications into their active or inactive forms. Small differences in the genetic sequences of these enzymes can greatly impact a drug's safety or effectiveness.

\section{Research focus}

More powerful medicines with greater targeting ability for specific diseases while decreasing damage to nearby healthy cells is just one advantage of using pharmacogenomics. Recovery times are going down and safety is being increased as the likelihood of adverse reactions goes down or is eliminated altogether. Current methods of basing dosages on weight and age are being replaced with dosages based on a person's genetics and how well the body processes the medicine and the time it takes to metabolize it.

Eventually it can be anticipated that the use of pharmacogenomics will lead to an overall decrease in the cost of healthcare because of decreases in several stakeholder areas: number of failed drug trials, the number of medications a patient must take to find an effective therapy, the time it takes to get new drugs approved, the length of time
Volume 4 Issue 5 - 2016

\author{
Charles D Shively \\ Chief Executive Healthcare Officer, USA
}

Correspondence: Charles D Shively, The Healthcare Advocate, Chief Executive Healthcare Officer, St. Petersburg, Florida, USA, Email charles@pharmacyce.com

Received: December 01, 2015 | Published: October 19, 2016

patients are on the medication and of course the number of adverse drug reactions.

The Precision Medicine Initiative Cohort Program (PMI) was announced by President Obama during his State of the Union address to the US public in January of 2015. It is a program that the National Institutes of Health $(\mathrm{NIH})$ will administer following expected United States Congressional funds appropriation in early 2016. PMI will enable a new era of medicine in which researchers, providers and patients work together to develop individualized care. A key component of this program is the involvement through clinical research of one million or more Americans from various ethnicities and backgrounds to allow extension of pharmacogenetics to many diseases past those studied to date. The framework for this large effort was approved by NIH in September of 2015 with a planned \$130million US to NIH including an additional \$70million US to the National Cancer Institute to lead cancer genomics as part of PMI for Oncology. Interestingly, a similar national cohort platformin the United States has already initiated for the study of Alzheimer's disease with a goal of issuing final recommendations for treatment guidelines by 2020 .

With the wide spread adoption of electronic health records, genomic analysis costs lowering, increased sophistication of data science and mobile health technologies, this PMI will be a participant-centered, data-driven enterprise with involving human biology, behavior, genetics, environment, data science and computation product new knowledge which supports effective ways to prolong health and treat disease.

Currently much of the research in this field is focused on genes encoding of either metabolic enzymes that can alter a drug's activity or defective structural proteins that result in increased susceptibility to disease.

\section{Research results}

The Clinical Pharmacogenetics Implementation Consortium (CPIC), the Royal Dutch Association for the Advancement of Pharmacy-Pharmacogenetics Work Group (DPWG) and other professional societies have made available Dosing Guidelines for 
some 35 drugs known to show variability in treatment success for various diseases based upon genome-disease associations. www. pharmgkb.org.

As specific examples, research has shown that the chemotherapy drugs cetuximab (Erbitux) and panitumumab (Vecitibix) do not work very well in 40 percent of colon cancer patients whose tumors have a particular genetic change. Recently researchers identified genetic variations that influence the response of people with clinically diagnosed depression to citalopram (Celexa) which belongs to a class of antidepressant drugs called selective serotonin re-uptake inhibitors (SSRIs).Pharmacogenomics allowed definition that the breast cancer drug trastuzumab (Herceptin) only works for women whose tumors have a particular genetic profile that leads to overproduction of a specific protein called HER2. Currently much of the research in this field is focused on genes encoding either metabolic enzymes that can alter a drug's activity or defective structural proteins that result in increased susceptibility to disease.

The United States Food and Drug Agency (FDA) have allowed pharmacogenomics information to be included in label insert copy for some 150 medications. This awareness, once genetic testing is completed on the patient, allows prescribing physicians and dispensing pharmacists to agreeonindividualized drug therapy, decreasing or lessening the chance for adverse drug events, and increasing the dose specific effectiveness of the drug.

\section{Global personalized therapeutics harmonization}

With the forthcoming PMI effort to enroll one million or more participants of varied ethnicities living in the United States, this platform project will no doubt lead to worldwide sharing of information and supportive research in the field of pharmacogenomics. The identification of the genetically-based drivers of disease in order to develop new, more effective treatments should support current World Health Organization goals.

\section{Acknowledgements}

None.

\section{Conflict of interest}

Author declares that there is no conflict of interest. 